ARTÍ́CULO ORIGINAL-CARDIOLOGÍA DEL ADULTO

\title{
Prevalencia de obesidad y riesgo cardiovascular en trabajadores del área de hidrocarburos
}

\section{Prevalence of obesity and cardiovascular risk in workers in the hydrocarbon area}

\author{
Edwards A. Velandia-Sua ${ }^{1,2 *}$, Ledmar J. Vargas-Rodríguez ${ }^{3}$, Óscar A. Vargas-Gil ${ }^{4}$ y \\ Hernando A. Benavidez-Jiménez ${ }^{5}$

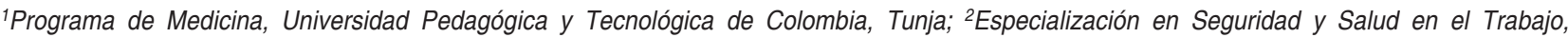 \\ Universidad de Boyacá, Tunja; ${ }^{3}$ Programa de Medicina, Universidad de Boyacá, Tunja; ${ }^{4}$ Programa de Medicina, Universidad Nacional de Colombia, \\ Bogotá; ${ }^{5}$ Programa de Medicina, Universidad de Ciencias Aplicadas y Ambientales, Bogotá. Colombia
}

\section{Resumen}

Introducción: La enfermedad cardiovascular (infarto de miocardio, muerte cardíaca y accidente cerebrovascular fatal o no fatal) es la principal causa de muerte prematura y de discapacidad en el mundo. Es responsable de más de 17 millones de muertes anuales y afecta principalmente a la población que se ubica en los países de ingresos bajos y medios. Se estima que el $80 \%$ de la carga mundial se produce en estos países. Objetivo: Establecer la prevalencia de obesidad y riesgo cardiovascular mediante los criterios de Framingham en una población de trabajadores de la industria de producción de hidrocarburos. Método: Se desarrolló un estudio observacional descriptivo, epidemiológico, en el que se tuvo en cuenta la población de los trabajadores de la empresa Montajes JM, Cerritos CPE6, Proyecto 176, en el departamento del Meta (Puerto Gaitán). Se representaron las variables en promedio, intervalos de confianza y desviación estándar, procediendo a desarrollar un análisis por sexo para cada uno de los factores de riesgo. Resultados: En la población del programa de riesgo cardiovascular se encontró un promedio de edad de 38.11 años y un índice de masa corporal de $26.00 \mathrm{~m}^{2} / \mathrm{kg}$. El $7.58 \%$ presentó hipertensión arterial, el 1.08\% diabetes mellitus y el $24.91 \%$ tabaquismo. El riesgo cardiovascular calculado con la escala de Framingham fue del 4.48\%. El índice de masa corporal, la edad y la presión arterial sistólica no son estadísticamente significativos para la valoración del riesgo con la escala Framingham. Conclusiones: La prevalencia de obesidad fue del $64.9 \%$, en tanto que el $75 \%$ de las personas que trabajan en hidrocarburos tenían riesgo leve de enfermedad cardiovascular.

Palabras clave: Escala de Framingham. Riesgo cardiovascular. Prevalencia. Hipertensión arterial. Salud pública.

\section{Abstract}

Introduction: Cardiovascular disease is one of the main causes of premature death and disability worldwide (myocardial infarction, cardiac death and fatal or non-fatal stroke) is responsible for more than 17 million annual deaths, in patients of low- and middle-income countries, it is estimated that $80 \%$ of the global burden occurs in these countries. Objective: To establish the prevalence of obesity and cardiovascular risk, evaluated using the Framingham criteria in a population of workers in the hydrocarbon production industry. Method: An observational, descriptive, epidemiological study was carried out of a population of workers of the company Montajes JM, Cerritos CPE6 Project 176, in Puerto Gaitan in the department of Meta,

\section{Correspondencia:}

*Edwards A. Velandia-Sua

E-mail: edanvesu2009@ hotmail.com
Fecha de recepción: 01-07-2020

Fecha de aceptación: 03-11-2020

DOI: 10.24875/RCCAR.M22000118
Disponible en internet: 22-02-2022

Rev Colomb Cardiol. 2022;29(1):57-63

www.rccardiologia.com

0120-5633 / @ 2020 Sociedad Colombiana de Cardiología y Cirugía Cardiovascular. Publicado por Permanyer. Este es un artículo open access bajo la licencia CC BY-NC-ND (http://creativecommons.org/licenses/by-nc-nd/4.0/). 
Colombia. The variables in average, standard deviation and confidence intervals were described, with a comparative analysis of risk factors by sex. Results: In the population of the cardiovascular risk program, an average age of 38.11 years old and a body mass index of $26.00 \mathrm{~m}^{2} / \mathrm{kg}$ were found. $7.58 \%$ presented high blood pressure, $1.08 \%$ diabetes mellitus and $24.91 \%$ were found to smoke. The cardiovascular risk calculated with the Framingham scale was found to be $4.48 \%$. Body mass index, age and systolic blood pressure were not statistically significant for the risk assessment using the Framingham scales. Conclusions: The prevalence of obesity in the group of workers in the hydrocarbon drilling industry was $64.9 \%$ and $75 \%$ had a slight risk of cardiovascular disease.

Keywords: Framingham scale. Cardiovascular risk. Prevalence. Arterial hypertension. Public health.

\section{Introducción}

Las muertes por enfermedades cardiovasculares han aumentado en el mundo debido al crecimiento poblacional, al envejecimiento y a los cambios epidemiológicos $^{1}$. Adicionalmente, la enfermedad cardiovascular es la principal causa de muerte prematura y de discapacidad en el mundo (infarto de miocardio, muerte cardíaca y accidente cerebrovascular fatal o no fatal), y es responsable de más de 17 millones de muertes anuales, con lo que se afecta principalmente a la población que se ubica en los países de ingresos bajos y medios; se estima que el $80 \%$ de la carga mundial se produce en estos países².

El estudio Framingham y diferentes estudios epidemiológicos de cohorte permitieron un cambio de enfoque, estadificación e identificación del riesgo cardiovascular en el siglo xx. Las investigaciones desarrolladas en este período ayudaron a dilucidar factores de riesgo cardiovascular, como edad, peso, sexo, hipertensión, hiperlipidemia, diabetes mellitus, colesterol total o número de cigarrillos fumados ${ }^{3,4}$.

El área de trabajo suele ser el primer sitio donde se desarrollan y se manifiestan factores de riesgo que pueden ir en detrimento de la salud de los trabajadores, y en los que se puede intervenir para realizar promoción de la salud y prevención de la enfermedad; sin embargo, las actividades de la industria del gas y el petróleo se llevan a cabo en sitios distantes, que producen cambios en los hábitos de vida y dificultan el acceso a la atención médica especializada. Una de las principales causas que activa el Medevac (medical evacuation) son los infartos agudos de miocardio y los accidentes cerebrovasculares, los cuales han aumentado en incidencia durante los últimos años en esta población ${ }^{5}$.

Adicionalmente, se ha evidenciado que las personas que se exponen a este tipo de trabajo tienen la necesidad de realizar labores nocturnas, lo cual se asocia con un incremento del riesgo de enfermedad coronaria, entre lo que se atribuye a un aumento de estrés laboral, conflictos familiares, problemas personales, consumo de tabaco y cambios en el ritmo circadiano ${ }^{6-8}$. A pesar de esto, son pocas las empresas que tienen programas bien estructurados para prevenir este tipo de enfermedades, ya sea porque creen que no se relacionan con la productividad o porque consideran que es responsabilidad exclusiva del trabajador y no les interesa su calidad de vida ${ }^{9}$. Por este motivo se realizó esta investigación, que tiene como objetivo analizar el riesgo cardiovascular de trabajadores de operaciones petroleras.

\section{Método}

Se ejecutó un estudio descriptivo entre el 1 de enero y el 1 de marzo de 2020, en el que la muestra estaba constituida por toda la población trabajadora de la empresa Montajes JM, cuya operadora es Frontera Energy, ubicada en Puerto Gaitán, Meta.

La muestra estuvo constituida por la totalidad del personal que se encontraba en la planta en el momento del estudio ( $n=350)$, todas las personas que tuvieran contrato laboral con la empresa y que en su historial clínico tuvieran reportados los registros clínicos de ingreso que permitieran aplicar la escala de Framingham (edad, índice de masa corporal [IMC], presión arterial, perfil lipídico completo, diagnóstico de diabetes mellitus y tabaquismo). Se excluyeron aquellas personas externas a la empresa, trabajadores con registros médicos incompletos y personas que no firmaran el consentimiento informado.

Las variables que se incluyeron correspondían a los factores de riesgo cardiovascular: edad, presión arterial, talla, peso, IMC, colesterol total, triglicéridos, colesterol unido a lipoproteínas de alta densidad (HDL) y glucemia. Se precisaron conceptos como hipertensión arterial (presión arterial > 140/90 mmHg, o diagnóstico previo o uso de medicación), sobrepeso (IMC > 25-29.9), obesidad (IMC > 30), colesterol total limítrofe alto (entre 200 y 239), colesterol alto (> 240), colesterol HDL bajo ( $40 \mathrm{mg} / \mathrm{dl}$ ), triglicéridos limítrofes altos (entre $150 \mathrm{y}$ 
$199 \mathrm{mg} / \mathrm{dl}$ ), triglicéridos altos (entre 200 y $249 \mathrm{mg} / \mathrm{dl}$ ) y muy altos (> $500 \mathrm{mg} / \mathrm{dl}$ ), hiperlipidemia (considerada como la combinación de los siguientes conceptos: colesterol total limítrofe alto o triglicéridos limítrofes altos) ${ }^{6}$ y diabetes mellitus (glucemia basal $>126 \mathrm{mg} / \mathrm{dl}$ o poscarga $>200)^{7}$. Se determinó la prevalencia incluyendo los factores de riesgo, con lo cual se realizó el cálculo del riesgo cardiovascular según Framingham, teniendo en cuenta los criterios descritos en la literatura $^{4}$ : $\sin$ riesgo, $<1 \%$; riesgo leve, $1-10 \%$; riesgo intermedio, $10-20 \%$; y riesgo alto o muy alto, $>20 \%$.

Los datos fueron recolectados por dos investigadores, quienes se encargaban de realizar la consulta médica al personal de trabajo, les explicaban la finalidad del estudio (riesgos y beneficios), entregaban el consentimiento informado para la respectiva firma de autorización de participación y diligenciaban un formulario con las variables de interés del estudio (mencionadas anteriormente). Luego se creó una base de datos en Excel y se realizó el análisis estadístico en SPSS versión 22. El análisis univariado de la población escogida se hizo estableciendo frecuencias absolutas y relativas para las variables categóricas, en tanto que para las variables cuantitativas se realizaron el cálculo de tendencia central (media, mediana) y medidas de dispersión según la distribución de la variable.

Apoyados en la Resolución 8430 de 1993, en la cual se instauran las normas de investigación en salud, se supone una investigación sin riesgo, en la que se recabó el debido consentimiento informado para la revisión del historial clínico del personal de la empresa.

\section{Resultados}

Del total de los trabajadores se excluyeron 73 personas: 51 no aceptaron la participación en el estudio, 5 laboraban en una planta distinta y 17 no se realizaron los exámenes de laboratorio, por lo cual tuvieron historias clínicas incompletas. En total, se incluyeron 277 personas que se encontraban laborando en la planta Cerritos CPE6 del proyecto 176 de la empresa Montajes JM.

Del total de participantes, un $96.03 \%$ fueron hombres, con un promedio de edad poblacional de 38.11 años (desviación estándar: \pm 10.32 ). En las variables de medidas antropométricas se halló un peso promedio de $73.9 \mathrm{~kg}$ y una talla promedio de $168 \mathrm{~cm}$; del IMC se computó una media estadística de $26.00 \mathrm{~m}^{2} / \mathrm{kg}$ (Tabla 1).

Entre los trabajadores incluidos en el programa, $21(7.58 \%)$ eran hipertensos crónicos y $3(1.08 \%)$ tenían diabetes mellitus (Tabla 2). Para las variables que incluían análisis del perfil lipídico se encontró que el
Tabla 1. Variables cuantitativas de la población

\begin{tabular}{|l|c|c|}
\hline Variable & Media \pm DE & Mínimo-máximo \\
\hline Edad (años) & $38.12 \pm 10.32$ & $36.90-39.33$ \\
\hline Peso $(\mathrm{kg})$ & $73.90 \pm 11.78$ & $72.51-75.28$ \\
\hline Talla $(\mathrm{cm})$ & $1.68 \pm 0.068$ & $1.67-1.68$ \\
\hline IMC $\left(\mathrm{kg} / \mathrm{m}^{2}\right)$ & $26.08 \pm 3.536$ & $25.67-26.50$ \\
\hline Colesterol HDL $(\mathrm{mg} / \mathrm{dl})$ & $45.74 \pm 10.11$ & $44.55-49.93$ \\
\hline Colesterol total $(\mathrm{mg} / \mathrm{dl})$ & $200.50 \pm 41.48$ & $195.61-205.38$ \\
\hline Presión diastólica $(\mathrm{mmHg})$ & $119.9 \pm 9.626$ & $118.76-121.03$ \\
\hline
\end{tabular}

DE: desviación estándar; HDL: lipoproteínas de alta densidad; IMC: índice de masa corporal.

Tabla 2. Variables cualitativas de la población

\begin{tabular}{|l|l|l|l|}
\hline Variable & \multicolumn{2}{|l|}{ Frecuencia (n) } & Porcentaje \\
\hline Sexo & Masculino & 266 & 96.03 \\
\hline & Femenino & 11 & 3.97 \\
\hline Diabetes mellitus & Sí & 3 & 1.08 \\
\hline Consumo de cigarrillo & So & 274 & 98.92 \\
\hline Hipertensión arterial & Sí & 69 & 24.91 \\
\hline & No & 208 & 75.09 \\
\hline & No & 21 & 7.58 \\
\hline
\end{tabular}

colesterol HDL mostró una media estadística de $45.74 \mathrm{mg} / \mathrm{dl}$, la cual estaría en rangos normales, y el colesterol total presentó una media estadística de $200.50 \mathrm{mg} / \mathrm{dl}$, levemente por encima de los rangos de normalidad lipídica; los valores fueron tomados de la guía de la Sociedad Europea de Cardiología (Tabla 1).

En cuanto a la presión arterial sistólica, se halló una media de $119 \mathrm{~mm} \mathrm{Hg}$, que se encuentra dentro de la categorización óptima; estos valores fueron tomados de la Sociedad Europea de Hipertensión ESH/ESC18 (Tabla 1).

De los 277 trabajadores, el $64.9 \%$ tenía un IMC elevado $(52.3 \%$ sobrepeso y $12.6 \%$ obesidad) (Tabla 3$)$.

En la tabla 4 se muestran los resultados del riesgo cardiovascular según la escala de Framingham. La población estudiada, según la mencionada escala, exhibe una media estadística de $4.48 \%$, valor que corresponde a riesgo leve; la población que se encuentra en 
Tabla 3. Prevalencia de obesidad

\begin{tabular}{|l|c|c|c|c|c|}
\hline $\begin{array}{l}\text { Características } \\
\text { sociodemográficas }\end{array}$ & Normopeso $(\mathbf{n}=\mathbf{9 7})$ & Sobrepeso $(\mathbf{n}=\mathbf{1 4 5})$ & Obesidad $(\mathbf{n}=\mathbf{3 5})$ & Total $(\mathbf{n}=\mathbf{2 7 7})$ & Mínimo-máximo \\
\hline $\begin{array}{l}\text { Sexo } \\
\text { Femenino } \\
\text { Masculino }\end{array}$ & $4(36.36 \%)$ & $7(63.63 \%)$ & 0 & $11(3.97 \%)$ & $56.31-70.29$ \\
\hline $\begin{array}{l}\text { Comorbilidad } \\
\text { Hipertensión arterial }\end{array}$ & $93(34.96 \%)$ & $138(51.87 \%)$ & $35(13.15 \%)$ & $266(96.02 \%)$ & $72.88-75.72$ \\
\hline $\begin{array}{l}\text { Tabaquismo } \\
\text { Diabetes }\end{array}$ & $7(33.33 \%)$ & $10(47.61 \%)$ & $4(19.04 \%)$ & $21(7.58 \%)$ & $118.76-121.03$ \\
\hline & $23(33.33 \%)$ & $38(55.07 \%)$ & $8(11.59 \%)$ & $69(24.90 \%)$ & \\
\end{tabular}

Tabla 4. Cálculo del riesgo cardiovascular según la escala de Framingham

\begin{tabular}{|lc|}
\hline Promedio $\pm \mathrm{DE}$ & $4.48 \pm 6.12$ \\
\hline IC95\% & 3.76 \\
\hline Riesgo leve (\%) & 75.09 \\
\hline Riesgo moderado (\%) & 24.91 \\
\hline Riesgo alto (\%) & 0 \\
\hline Riesgo muy alto (\%) & 0 \\
\hline
\end{tabular}

DE: desviación estándar; IC95\%: intervalo de confianza del 95\%.

riesgo bajo corresponde al $75.09 \%$, en riesgo moderado se encuentra el $24.91 \%$ de la población, mientras que en los otros dos niveles de riesgo no se encuentra ningún trabajador.

En la tabla 5 se muestran la comparación del riesgo por sexos, las variables antropométricas y el perfil lipídico, los cuales presentaron diferencia estadísticamente significativa. El IMC evidencia que la mayoría de los participantes se encuentran en sobrepeso, pero su valor está aumentado en los hombres ( $p=0.2910)$; la variable colesterol HDL se encuentra en valores normales para hombres y mujeres $(p=0.0000)$, y la presión arterial sistólica fue normal para ambos sexos. Los valores guía fueron tomados de la Sociedad Europea de Cardiología.

En la tabla 6 se muestra el cálculo del riesgo cardiovascular por sexo según la escala de Framingham. No hay diferencia estadísticamente significativa entre los sexos; según esta escala, el riesgo fue bajo para hombres y mujeres $(p=0.0000)$.

\section{Discusión}

Al definir el riesgo cardiovascular en trabajadores de la industria de producción de hidrocarburos se encontró que el riesgo cardiovascular calculado fue del $4.48 \%$ según la escala de Framingham, lo que se traduce en que la mayoría de los trabajadores tienen riesgo leve. Según Álvarez et al. ${ }^{10}$, el riesgo cardiovascular para pacientes pertenecientes a un primer nivel de atención en la ciudad de Armenia, Quindío, determinado por la escala de Framingham, es del $11.36 \%$, cifra mayor que la obtenida en el presente estudio; sin embargo, cabe resaltar que la edad promedio en el estudio de Álvarez et al. ${ }^{10}$ fue de 64.54 años, mientras que en el nuestro fue de 38.11 años.

Por otro lado, con base en el estudio de Navarro y Rusvelt ${ }^{11}$ el riesgo cardiovascular en personas mayores con síndrome metabólico previo de la ciudad de Soledad, Atlántico, calculado según la escala de Framingham, fue del $3 \%$; es decir, riesgo bajo, dato que presenta similitudes con nuestros hallazgos. La edad promedio en los dos estudios es similar.

La población de hombres presentó un riesgo del $4.61 \%$ (riesgo bajo), frente al $1.03 \%$ en las mujeres (riesgo bajo). El riesgo encontrado es mayor en la población de hombres, teniendo en cuenta que la población de hombres corresponde al $96.02 \%$, con un promedio de 38.11 años. Navarro y Rusvelt ${ }^{11}$ determinaron que el riesgo cardiovascular es mayor en la población masculina $(5.67 \%)$ y que las mujeres tienen un riesgo menor (1.01\%). Aun así, los dos grupos poblacionales se clasifican en riesgo bajo. Se debe considerar que la población que más se dedica a los trabajos de la industria del petróleo y los hidrocarburos pertenece al sexo masculino y, por tanto, tiene más posibilidad de presentar riesgo cardiovascular; de ahí que sea importante hacer un estricto control y seguimiento en cuanto a sus hábitos de vida ${ }^{12}$.

La hipertensión arterial tiene una prevalencia del $7.58 \%$ según los hallazgos de nuestro estudio. Por su parte, en una revisión sistemática y metaanálisis, Zurique et al. ${ }^{13}$ encontraron una prevalencia de hipertensión arterial del $24 \%$ en los 23 estudios incluidos, con 
Tabla 5. Comparación de riesgo entre los sexos

\begin{tabular}{|l|c|c|c|c|c|}
\hline Variable & Masculino Media \pm DE & Mínimo-máximo & Femenino Media \pm DE & Mínimo-máximo & $\mathbf{p}\left(\chi^{2}\right)$ \\
\hline Peso $(\mathrm{kg})$ & $74.3 \pm 11.78$ & $72.88-75.72$ & $63.3 \pm 11.82$ & $56.31-70.29$ & 0.0110 \\
\hline Talla $(\mathrm{cm})$ & $1.69 \pm 0.068$ & $1.68-1.70$ & $159 \pm 0.06$ & $158.96-159.04$ & 0.0020 \\
\hline IMC $\left(\mathrm{kg} / \mathrm{m}^{2}\right)$ & $26.1 \pm 3.536$ & $25.68-26.52$ & $25.1 \pm 3.51$ & $23.02-27.18$ & 0.2910 \\
\hline Edad (años) & $38.2 \pm 10.32$ & $36.99-39.44$ & $36 \pm 10.95$ & $29.74-42.26$ & 0.8790 \\
\hline Colesterol HDL $(\mathrm{mg} / \mathrm{dl})$ & $45.16 \pm 10.11$ & $43.95-46.37$ & $59.72 \pm 10.01$ & $53.81-65.63$ & 0.0000 \\
\hline Colesterol total $(\mathrm{mg} / \mathrm{dl})$ & $199.7 \pm 41.48$ & $194.71-204.69$ & $219 \pm 41.33$ & $194.57-243.43$ & 0.0000 \\
\hline Presión diastólica $(\mathrm{mmHg})$ & $119.96 \pm 9.626$ & $118.80-121.19$ & $118 \pm 9.66$ & $112.29-123.71$ & 1.0000 \\
\hline
\end{tabular}

DE: desviación estándar; HDL: lipoproteínas de alta densidad; IMC: índice de masa corporal.

Tabla 6. Comparación del riesgo entre los sexos

\begin{tabular}{|l|c|c|c|c|}
\hline Cálculo del riesgo Framingham & Masculino Media \pm DE & Mínimo-máximo & Clasificación del riesgo & $\mathrm{p}\left(\chi^{2}\right)$ \\
\hline & $4.61 \pm 6.117$ & $3.934-5.404$ & Bajo & 0.0000 \\
\hline & Femenino media \pm DE & Mínimo-máximo & Clasificación del riesgo & $\mathbf{p}\left(\chi^{2}\right)$ \\
\hline
\end{tabular}

DE: desviación estándar.

una población de 52,570 individuos. El Plan Decenal en Salud de Colombia (2012-2021) define, para la población del país, una prevalencia de hipertensión arterial del $22.8 \%$, lo que indica que hay una asociación con los pacientes que presentan cardiopatía isquémica, en un $49 \%$, y con aquellos que presentan accidentes cerebrovasculares, en un $62 \%{ }^{14}$.

La prevalencia de diabetes fue del $1.08 \%$ en este estudio. Según el Plan Decenal de Salud en Colombia, se encontró que la prevalencia de diabetes mellitus fue del $2.6 \%$ en el año $2007^{14}$. Específicamente para Colombia, según el Atlas de Diabetes de la Federacion Internacional de la Diabetes (FID), el número de personas con diabetes mellitus tipo 2 fue de 2,135,380, con una prevalencia reportada del $7.12 \%$, en el grupo etario entre los 20 y 79 años, para el año 2013 $3^{15-20}$.

Por otro lado, la prevalencia de tabaquismo en el mundo es variable: va desde el $48.6 \%$ (intervalo de confianza del 95\% [IC95\%]: 47.6-49.6) en hombres hasta el $11.3 \%$ (IC95\%: 10.7-12.0) en mujeres. EI Plan Decenal en Salud de Colombia refiere una prevalencia del $12.8 \%$ en el consumo de tabaco en $2007^{14}$. Para Álvarez et al. ${ }^{10}$, en el estudio de riesgo cardiovascular se evidencia un porcentaje de fumadores del $9.36 \%$.
Los trabajadores estudiados tienen un IMC calculado con una media de $26.08 \mathrm{~m}^{2} / \mathrm{kg}$, en sobrepeso; la prevalencia de sobrepeso y obesidad para ambos sexos fue del $64.29 \%$. Durante el año 2010 se dieron a conocer los resultados de la Segunda Encuesta Nacional de la Situación Nutricional en Colombia (ENSIN), que indican una prevalencia de sobrepeso y obesidad entre los colombianos del $51.2 \%$, entre los 18 y los 64 años $)^{17}$. Por su parte, Álvarez et al. ${ }^{10}$ determinaron que el IMC fue de $27.71 \mathrm{~m}^{2} / \mathrm{kg}$, es decir, sobrepeso. El IMC como factor de riesgo no fue concluyente en la escala de Framingham ( $p=0.2910)$.

El colesterol HDL evidenció una media de $45.74 \mathrm{mg} /$ $\mathrm{dl}$, que corresponde a valores dentro de la normalidad para la población, con un valor promedio de $45.16 \mathrm{mg} /$ dl para los hombres y de $59.72 \mathrm{mg} / \mathrm{dl}$ para las mujeres, hallazgos que, cruzados con el tercer Informe del Panel de Expertos en Detección, Evaluación y Tratamiento del Colesterol Alto en Sangre en Adultos, se hallan en metas $^{19}$. La presión arterial sistólica evidenció una media de $119 \mathrm{mmHg}$ (óptima), con una prevalencia poblacional del $7.58 \%$. Para Lombo et al..$^{18}$, la presión arterial sistólica media fue de $126.35 \mathrm{mmHg}$. De acuerdo con los estudios realizados por Aristizábal Ocampo ${ }^{21}$, la 
hipertensión tiene un riesgo atribuible del 32.3\%. Cuando se determinaron los factores de riesgo en la escala de Framingham, se observó que algunas variables no fueron estadísticamente significativas, como el IMC, la edad y la presión arterial sistólica, que anteriormente en otros estudios evidenciaban estas características de la escala para la población colombiana ${ }^{10}$.

Tenkanen et al. ${ }^{22}$, en un estudio de cohorte sobre una población de 1806 trabajadores, encontraron que todos los trabajadores en turnos nocturnos, comparados con los trabajadores de día, tenían un riesgo relativo de enfermedad cardiovascular de 1.52 (IC95\%: 1.11-2.07). Así mismo, reportaron que los trabajadores de campo de día, de campo en dos turnos y de campo en tres turnos tuvieron un riesgo relativo para enfermedad cardiovascular de 1.3 (IC95\%: 0.8-2.0), 1.9 (IC95\%: 1.1-3.4) y 1.7 (IC95\%: 1.1-2.7), respectivamente, comparados con los trabajadores de oficina que hacian solo turnos de día. Datos similares a los mencionado anteriormente fueron reportados por Virkkunen et al. ${ }^{23}$ y Tuchson et al. ${ }^{24}$, quienes encontraron una asociación entre los trabajos nocturnos por más de 5 años como factor relacionado con el riesgo cardiovascular.

Palacios Ruesta ${ }^{25}$ reportó que en los trabajadores de plataformas petroleras no aumenta la frecuencia de los factores de riesgo cardiovascular, pero sí hay un incremento en el nivel de riesgo de Framingham, así como un mayor porcentaje de trabajadores con alto riesgo cardiovascular según Framingham (12,5\% y 6,3\%).

A pesar de ello, es de vital importancia tener presente el estudio realizado por López Puerto ${ }^{26}$, quien concluye que en las personas que laboran en operaciones petroleras la implementación de programas preventivos para el riesgo cardiovascular tiene un buen impacto, ya que genera cambios en los hábitos de vida y, a su vez, estos se transmiten a sus familiares y amigos, con lo que contribuyen a la disminución del riesgo de presentar infarto agudo de miocardio y otras enfermedades cardiovasculares.

Una de las limitaciones del estudio es que se realizó una entrevista a los participantes, en la que varios pueden dar respuestas que no concuerdan con la realidad; adicionalmente, es de vital importancia tener en cuenta que, hasta el momento, no se han reportado estudios en los que se evalúe este tipo de población, lo que genera dificultad en la comparación de los hallazgos.

\section{Conclusiones}

En este estudio fue posible determinar la prevalencia de obesidad: el $64.9 \%$ de los participantes tenían un
IMC elevado, el $52.3 \%$ de los cuales estaban en sobrepeso y el $12.6 \%$ eran obesos. A pesar de ello, se encontró que el $75 \%$ de los participantes se encontraban en riesgo cardiovascular leve.

\section{Agradecimientos}

Los autores agradecen a los participantes, por su buena disposición y colaboración en la presente investigación.

\section{Financiamiento}

Los autores declaran no haber recibido financiamiento.

\section{Conflicto de intereses}

Los autores no presentan conflictos de intereses.

\section{Responsabilidades éticas}

Protección de personas y animales. Los autores declaran que para esta investigación no se han realizado experimentos en seres humanos ni en animales.

Confidencialidad de los datos. Los autores declaran que en este artículo no aparecen datos de pacientes.

Derecho a la privacidad y consentimiento informado. Los autores declaran que en este artículo no aparecen datos de pacientes.

\section{Bibliografía}

1. Roth GA, Forouzanfar MH, Moran AE, Barber R, Nguyen G, Feigin VL, et al. Demographic and epidemiologic drivers of global cardiovascular mortality. N Engl J Med. 2015;372:1333-41.

2. Du H, Li L, Bennett D, Guo Y, Key YJ, Phil D, et al. Fresh fruit consumption and major cardiovascular disease in China. $\mathrm{N}$ Engl $\mathrm{J}$ Med. 2016;374:1332-43

3. Mahmood SS, Levy D, Vasan RS, Wang TJ. The Framingham Heart Study and the epidemiology of cardiovascular disease: a historical perspective. Lancet. 2014;383:999-1008.

4. Goodarz D, Yuan L, Gitanjali MS, Emily C, Gretchen A. Cowan ME, et al. Cardiovascular disease, chronic kidney disease, and diabetes mortality burden of cardiometabolic risk factors from 1980 to 2010: a comparative risk assessment. Lancet Diabetes Endocrinol. 2014;2:634-47.

5. Organización Internacional del Trabajo. La seguridad y salud en el trabajo y las necesidades en materia de competencias profesionales en la industria del petróleo y el gas que opera en zonas climáticas polares y subárticas del Hemisferio Norte. Informe para la discusión en la reunión sectorial tripartita sobre la seguridad y salud en el trabajo y las necesidades en materia de competencias profesionales en la industria del petróleo y el gas que opera en zonas climáticas polares y subárticas del Hemisferio Norte (Ginebra, 26-29 de enero de 2016). Disponible en: https://www.ilo.org/wcmsp5/groups/public/---ed_dialogue/---sector/documents/publication/wcms_438076.pdf.

6. Costa G. Shift work and health: current problems and preventive actions Obra sanitaria saf. 2010 diciembre: 1(2): 113 - 123.

7. Harrington JM. Health effect of shift work and extended hours of work. Occup Environ Med. 2001;58:68-72. 
8. van Amelsvoort LG, Schouten EG, Kok FJ. Impact of one year of shift work on cardiovascular disease risk factors. J Occup Environ Med. 2004;46:699-706.

9. Spurgeon A, Harrington JM, Cooper CL. Health and safety problems associated with long working hours. Occup Environ Med. 1997:54:367-75.

10. Álvarez J, Álvarez A, Carvajal W, González M, Duque J, Nieto O. Determinación del riesgo cardiovascular en una población. Rev Colomb Cardiol. 2017;24:334-41.

11. Navarro E, Rusvelt $F$. Riesgo coronario según ecuación de Framingham en adultos con síndrome metabólico de la ciudad de Soledad, Atlántico. 2010. Rev Colomb Cardiol. 2012;19:109-18.

12. Kane AE, Howlett SE. Differences in cardiovascular aging in men and women. Adv Exp Med Biol. 2018;1065:389-411.

13. Zurique M, Zurique $C$, Camacho $P$, Sánchez M, Hernández S. Prevalencia de hipertensión arterial en Colombia. Revisión sistemática y meta-análisis. Acta Med Colomb. 2019;44:1-15.

14. Gaviria A, Muñoz N, Ruiz F, Ospina M, Urquijo L, Arias J, et al. Plan Decenal de Salud Pública Min-Salud. 2013. p. 13-237. Disponible en: https://www. minsalud.gov.co/Documentos\%20y\%20Publicaciones/Plan\%20Decenal\%20 -\%20Documento\%20en\%20consulta\%20para\%20aprobaci\%C3\%B3n.pdf.

15. Vargas $H$, Casas L. Epidemiología de la diabetes mellitus en Sudamérica: la experiencia de Colombia. Clin Investig Arterioscler. 2016;28:245-56.

16. Giovino A, Mirza A, Samet J, Gupta P, Jarvis M, Bhala N, et al. Tobacco use in 3 billion individuals from 16 countries: an analysis of nationally representative cross-sectional household surveys. Lancet. 2012;380:668-79.

17. Ministerio de la Protección Social, Instituto Colombiano de Bienestar Familiar, Dirección de Prevención. Encuesta nacional de la situación nutricional en Colombia 2010. Revisado el 13 de mayo de 2020. Disponible en: https://www.minsalud.gov.co/sites/rid/Lists/BibliotecaDigital/ RIDE/VS/ED/GCFI/Base\%20de\%20datos\%20ENSIN\%20-\%20Protocolo\%20Ensin\%202010.pdf.
18. Lombo B, Villalobos C, Tique C, Satizábal C, Franco AC. Metabolic syndrome prevalence in patients attending the hypertension clinic at the Fundación Santa Fe de Bogotá. Rev Colomb Cardiol. 2006;12:472-8.

19. Expert Panel on Detection, Evaluation, and Treatment of High Blood Cholesterol in Adults. Executive Summary of the Third Report of the National Cholesterol Education Program (NCEP) Expert Panel on Detection, Evaluation and Treatment of High Blood Cholesterol in Adults (Adult Treatment Panel III). JAMA. 2001;285:2486-97.

20. Guías ALAD 2000 para el diagnóstico y manejo de la diabetes mellitus tipo 2 con medicina basada en evidencia. Revista de la Asociación Latinoamericana de Diabetes. 2000; Supl 1, Ed. Extraordinaria.

21. Aristizabal, D. La hipertensión en el Siglo XXI: Ios grandes cambios que nos esperan. Rev. Col. Cardiol. 2007, vol.14, n.4, pp.181-184.

22. Tenkanen L, Sjöblom T, Kalimo R, Alikoski T. Shift work occupation and coronary heart disease over 6 years of follow-up in the Helsinki Heart Study. Scand J Work Environ Health. 1997;23:257-65.

23. Virkkunen $\mathrm{H}$, Harma M, Kauppinen $\mathrm{T}$, Tenkanen $\mathrm{L}$. The triad of shift work, occupational noise, and physical workload and risk of coronary heart disease. Occup Environ Med. 2006;63:378-86.

24. Tuchson F, Hannerz H, Burr H. A 12 year prospective Study of Circulatory Disease Among Danish Shift Worker. Occup Envi-ron Med. 2006;63:451-5.

25. Palacios Ruesta RC. Determinación de los factores de riesgo cardiovascular en trabajadores a turnos en plataformas marítimas de una petrolera del Norte del Perú. Acta Med Per. 2011;28:67-72.

26. López Puerto IA. Efecto del programa de medicina preventiva bajo el sistema OHSAS 18001, sobre el riesgo cardiovascular en la Empresa Colombiana de Operaciones Petroleras. IGNOS. 2145-1389. 2014;6:59-72. 